\title{
Corps-machines à l'Opéra : l'imaginaire scientifique dans les écrits sur la danse (1830-1860)
}

"Body a Machine" at the Grand Opera: Scientific imagination in writings on dance (1830-1860)

\section{Bénédicte Jarrasse}

\section{(2) OpenEdition}

\section{Journals}

Édition électronique

URL : https://journals.openedition.org/aes/4075

DOI : $10.4000 /$ aes. 4075

ISSN : 2258-093X

Éditeur

Laboratoire LISAA

Référence électronique

Bénédicte Jarrasse, «Corps-machines à l'Opéra : l'imaginaire scientifique dans les écrits sur la danse (1830-1860) », Arts et Savoirs [En ligne], 16 | 2021, mis en ligne le 17 décembre 2021, consulté le 19 décembre 2021. URL : http://journals.openedition.org/aes/4075; DOI : https://doi.org/10.4000/aes. 4075

Ce document a été généré automatiquement le 19 décembre 2021.

Centre de recherche LISAA (Littératures SAvoirs et Arts) 


\title{
Corps-machines à l'Opéra : l'imaginaire scientifique dans les écrits sur la danse (1830-1860)
}

\author{
"Body a Machine" at the Grand Opera: Scientific imagination in writings on \\ dance (1830-1860)
}

Bénédicte Jarrasse

1 Le XIX ${ }^{e}$ siècle s'impose à bien des égards comme le siècle de la "dansomanie ", cette étrange maladie, attestée depuis la Renaissance, touchant des corps en proie à la folie dansante, qui donne son titre, de manière visionnaire, à un ballet de Pierre Gardel chorégraphié en $1800^{1}$. Il voit ainsi prospérer les bals, publics ou privés - événements inscrits dans une temporalité et une géographie urbaine et sociale bien définies -, et est en outre marqué par l'émergence et le succès européen du ballet romantique, phénomène qui s'accompagne d'une médiatisation inédite de l'art chorégraphique et de ses interprètes. La danse théâtrale, offerte à une multiplicité de regards au sein de la ville moderne, suscite alors des discours variés, aux contenus et aux enjeux parfois contradictoires, qui engagent, bien au-delà du champ traditionnel des professionnels de la discipline, la presse et, plus largement, les artistes - écrivains ou plasticiens.

2 Les observateurs - et commentateurs - de la révolution esthétique inaugurée par le romantisme sont, pour beaucoup, des personnalités extérieures à l'univers du ballet, des dilettantes et non-spécialistes revendiqués. Les feuilletonistes qui rendent régulièrement compte de l'actualité du ballet dans la presse et sont les principaux témoins de ses succès tendent de fait à appréhender le corps dansant de manière parcellaire - par ses à-côtés anecdotiques ou sa périphérie licencieuse - et détournée en l'euphémisant, en le fétichisant ou, au contraire, en l'essentialisant. Dès lors, il peut paraître curieux, voire paradoxal, d'invoquer le regard scientifique, ou pseudoscientifique, à propos d'une discipline souvent jugée futile, tout à la fois objet de fascination et de crainte. Ce serait pourtant faire fi des critiques des corps portées en creux par les comptes rendus de presse et, par ailleurs, de l'existence de discours - aux finalités certes distinctes - qui se présentent comme ouvertement désidéalisants : celui 
des physiologistes et des caricaturistes d'une part, qui, loin de se laisser abuser par les artifices du spectacle et les métamorphoses opérées par la scène, jettent, chacun à sa manière, un regard cru et volontiers désenchanté sur le quotidien des artistes et la mécanique des corps à l'œuvre dans les coulisses des théâtres, celui des pédagogues et des maîtres de ballet d'autre part, qui viennent rappeler, dans le cadre de traités théoriques ou pratiques, que la danse théâtrale est un " un art sérieux et compliqué »", qui s'appuie sur une technique savante, acquise au terme d'une formation longue et pénible, et relève à ce titre d'un travail, d'un entraînement, voire d'un "dressage ", paradoxalement destiné à effacer la part mécanique qu'elle comprend inévitablement. L'imaginaire du spectacle romantique peut dès lors se délecter - en l'exacerbant - de la tension d'un corps travaillé jusqu'à l'épuisement dans les coulisses des théâtres et produisant sur scène « l'exploit fantastique dont l'humanité vulgaire est incapable » .

La mise en lumière du "corps-machine", qu'elle soit critique, documentaire ou heuristique, favorise ainsi l'émergence, dans les années 1840-1850, d'un imaginaire de la danse, sinon scientifique, du moins teinté d'une scientificité inédite. Dans ce contexte, la danse n'est pas tant envisagée dans sa dimension spectaculaire, véhicule de l'émotion artistique, que comme une pratique et un métier, avant tout fondés sur la répétitivité du geste. Il s'agira donc de voir dans un premier temps comment cette approche nouvelle contribue à interroger et à remettre en question l'imaginaire enchanté $\mathrm{du}$ ballet, support de l'économie du vedettariat - du star-system - autour duquel se construit l'Opéra bourgeois du XIX ${ }^{e}$ siècle. Le corps romantique, dans sa version idéale, réelle ou fictionnelle, fascine en effet le spectateur pour autant qu'il parvient à se métamorphoser, à se dématérialiser, à se faire in fine oublier en tant que corps, dans ses dimensions à la fois anatomiques et mécaniques. Les représentations de corps, offertes aussi bien par les comptes rendus de spectacles que par les caricatures de presse, péchant tantôt par manque, tantôt par excès de chair ou de force musculaire, viennent dès lors définir un contre-modèle esthétique et moral, qui trouve sa justification savante dans la critique du corps-machine, présente dès le XvIII ${ }^{\mathrm{e}}$ siècle chez les théoriciens du ballet d'action.

Porteur d'une critique des corps, cet imaginaire se charge en outre de révéler au public une condition sociale, celle des danseuses du corps de ballet, dont le travail quotidien s'inscrit dans le contexte d'une société en voie d'industrialisation et de métamorphoses urbaines. L'opération de transfiguration, permise par la représentation scénique, n'est en effet possible qu'au prix d'un dressage des corps, dont font état de nombreuses physiologies et de publications anecdotiques traitant des coulisses des théâtres et en particulier de l'Opéra. Ce tableau, qui vulgarise un savoir sur le monde professionnel de la danse académique mais n'en est pas moins empreint de fantasmes, trouve à ce titre un écho et un prolongement " savant » dans le souci des pédagogues du milieu du siècle de rationaliser et de donner une base positive à l'art chorégraphique et, ainsi, dans un contexte de déploration générale autour du « déclin » de la danse, de le « régénérer ».

\section{La critique noverrienne du corps-machine}

5 Il nous semble nécessaire de revenir en préambule sur les conceptions développées au $\mathrm{XVIII}^{\mathrm{e}}$ siècle par l'un des spécialistes de la "chose" chorégraphique, Jean-Georges Noverre, théoricien et promoteur du ballet d'action, dans ses Lettres sur la danse ${ }^{4}$ conceptions qui déterminent en partie le regard romantique sur le corps dansant. 
L'enjeu du ballet d'action, qui perdure et s'accomplit au XIx ${ }^{e}$ siècle à travers les divers avatars du genre du ballet-pantomime, réside dans sa capacité à traduire par le geste par le seul véhicule du corps donc - l'essentiel d'une intrigue sans l'aide d'un livret. Autrement dit, le défi qui se pose à cette forme est celui de l'expressivité du corps dansant. Dans cette perspective, qui rejoint certains débats contemporains sur le théâtre, Noverre recourt à une image ancienne, celle du corps-machine, relayée notamment au XVIII ${ }^{\text {e }}$ siècle par La Mettrie, mais qu'on peut faire remonter à Descartes, voire à la Renaissance, au moment du développement de la science anatomique. Cette image s'inscrit dans une approche du mouvement inspirée du modèle de la machine, qui conditionne du reste, à l'époque de Noverre, la fabrication d'automates, androïdes ou animaliers, destinés à reproduire les mécanismes corporels, afin de mieux les comprendre et d'en améliorer les capacités ${ }^{5}$. Noverre reconnaît ainsi que « la danse de nos jours est belle » et que « l'exécution mécanique de cet art est portée à un degré de perfection qui ne laisse rien à désirer ", mais ajoute aussitôt :

Les pas, l'aisance et le brillant de leur enchaînement, l'aplomb, la fermeté, la vitesse, la légèreté, la précision, les oppositions des bras avec les jambes, voilà ce que j'appelle le mécanisme de la danse. Lorsque toutes ces parties ne sont pas mises en œuvre par l'esprit, lorsque le génie ne dirige pas tous ces mouvements, et que le sentiment et l'expression ne leur prêtent pas des forces capables de m'émouvoir et de m'intéresser, j'applaudis alors à l'adresse, j'admire l'homme-machine, je rends justice à sa force, à son agilité ; mais il ne me fait éprouver aucune agitation; il ne m'attendrit pas [...]..$^{6}$

Si le théoricien apparaît attentif à la puissance et à l'agilité dégagées par «l'homme machine» et souligne par ailleurs l'importance de l'étude anatomique dans l'amélioration des performances du danseur, la perfection technique ne doit selon lui jamais occulter l'émotion nécessairement attachée à l'interprétation :

Pour que notre art parvienne à ce degré de sublimité que je demande et que je lui souhaite, il est indispensablement nécessaire que les danseurs partagent leur temps et leurs études entre l'esprit et le corps et que tous les deux soient ensemble l'objet de leurs réflexions ; mais on donne malheureusement tout au dernier et l'on refuse tout à l'autre. La tête conduit rarement les jambes et, comme l'esprit et le génie ne résident pas dans les pieds, on s'égare souvent, l'homme s'éclipse, il n'en reste qu'une machine mal combinée, livrée à la stérile admiration des sots et au juste mépris des connaisseurs.

Étudions donc, Monsieur; cessons de ressembler à ces marionnettes dont les mouvements dirigés par des fils grossiers n'amusent et ne font illusion qu'au peuple.

7 Prônant le naturel dans l'expression, Noverre rejoint à travers ce propos des préoccupations qui touchent au même moment le monde théâtral dans son ensemble. Dans le contexte du ballet d'action, genre qui associe et fait alterner danse et pantomime, il s'agit de s'attaquer à une exécution purement mécanique du geste dansé, dans laquelle le corps humain se rapproche par analogie du corps artificiel d'un pantin ou d'une marionnette, simulacres d'humanité.

\section{Les corps dansants au cabinet d'anatomie}

8 L'image du corps-machine, empruntée à la philosophie des sciences, se retrouve ainsi au XIX ${ }^{e}$ siècle au cœur d'une vaste réflexion sur la nature du corps dansant, dont témoigne notamment le texte fondateur de Kleist, souvent cité, à travers la figure 
paradigmatique de l'automate ${ }^{8}$. Elle ressurgit en creux, avec des enjeux esthétiques et éthiques semblables, dans la réception critique des spectacles donnés à l'Académie royale de musique. Notons que les comptes rendus auxquels ceux-ci donnent lieu n'émanent pas de professionnels ou de spécialistes de l'art chorégraphique, mais d'hommes de lettres, simples amateurs de ballet, collaborant à tel ou tel périodique comme chroniqueurs théâtraux ou feuilletonistes. Le corps-machine, cantonné à une pure démonstration technique, à une virtuosité gratuite qui identifie l'interprète à un artiste sans âme, devient dès lors une cible de la critique journalistique et un topos de la caricature du corps dansant, qui s'explique par la crainte, non exempte de fascination, que celui-ci ne se réduise à la seule exhibition de tours de force. Si les prouesses physiques de l'acrobate, du funambule ou du danseur comique, tel le célèbre Mazurier ${ }^{9}$, au corps disloqué, sont grandement appréciées sur le Boulevard, dans les théâtres populaires voués au divertissement, comme la Gaîté, l'Ambigu-Comique ou la Porte Saint-Martin, elles sont ressenties comme inadéquates à l'Opéra, lieu institutionnel, emblématique depuis Louis XIV de la danse d'école.

9 Marie Taglioni est la danseuse qui contribue à l'Opéra, au début des années 1830, à redéfinir l'esthétique et la morale du corps dansant, alors corrompues, selon certains chroniqueurs, par les excès de la danse noble $e^{10}$. Avec elle, la danse ne repose plus sur la seule prouesse, mais sur des qualités plus abstraites et métaphysiques comme la grâce et la légèreté. En 1832, année de la création de La Sylphide, Jules Janin qualifie ainsi Albert, danseur réputé pour sa virtuosité dans les années 1810-1820, de « débris guindé et parfait de la danse noble », avant de le décrire comme " un pantin qui saute bien », une "machine ", tenue non par « un fil », mais par " un ressort » ${ }^{11}$. La partenaire du danseur noble, la danseuse noble donc, n'a rien à lui envier dans ce domaine et incarne de la même façon un style de danse reposant sur quelques effets spectaculaires - le saut et la pirouette finale "sur la pointe du pied », que Janin rebaptise la bouffante -, dont le public bourgeois de la monarchie de Juillet semble être lassé :

$\mathrm{Au}$ lieu de se précipiter comme fait le danseur, elle arrive à petits pas. Elle se pose comme sur le fil d'archal; elle commence par des jetés-battus, elle se baisse jusqu'à terre. Elle se relève et se baisse de nouveau; elle tourne le pied de côté et d'autre [...]. Mais peu à peu elle s'anime, elle sautille, elle fait plus que sautiller, elle saute tout à fait. - Et les bras roides, le corps immobile, la face souriante [...] !

Quand une danseuse noble a été en l'air noblement et qu'elle s'est noblement montrée sur toutes ses faces, le plus souvent de côté en penchant la tête, elle termine son assaut par une pirouette sur la pointe du pied. Cette pirouette commence vivement et va en s'affaiblissant; alors la robe de gaze de la déesse s'enfle comme un ballon [...]. ${ }^{12}$

Cette exécution mécanique, décrite en des termes imagés qui évoquent les caricatures de l'époque, s'oppose directement à l'idéal emblématisé par Taglioni, celui d'un corps léger, immatériel, sinon diaphane, produisant une danse naturelle et expressive, qui gomme toute sensation de dépense ou d'effort physique.

11 L'imaginaire mécaniciste n'est toutefois pas seul à véhiculer une critique des corps dansants. Gautier, par ailleurs grand admirateur de performances circassiennes et de virtuosité gratuite, l'approfondit en l'associant à un lexique anatomique dans un texte consacré aux danseurs espagnols invités à Paris dans le cadre du bal de l'Opéra. Il y oppose le danseur « ordinaire » - comprenons celui qu'il a coutume de voir évoluer sur la scène de l'Académie royale de musique - à ces fameux Espagnols, modèles de naturel, 
chez lesquels, a contrario des Français, «il n'y a rien de mécanique, rien d'emprunté et qui sente l'école $»^{13}$ :

Vous savez quelle chose hideuse c'est qu'un danseur ordinaire; un grand dadais avec un long cou rouge gonflé de muscles, un rire stéréotypé [...]; des yeux sans regard qui rappellent les yeux d'émail des poupées à ressort [...], de gros mollets de suisse de paroisse, des brancards de cabriolet en façon de bras, et puis de grands mouvements anguleux, les coudes et les pieds en équerre [...], des ronds de jambe, des pirouettes et autres gestes de pantins mécaniques. ${ }^{14}$

Pour littéraire et imagée qu'elle soit, la description est sans conteste informée par un savoir biologique et anatomique. L'hypertrophie des tendons et des muscles, associée à des gestes mécaniques et des mouvements anguleux évoquant la géométrie, produit un corps à la fois hybride et obscène. Dans la perspective " païenne » gautiériste, ce corps ne s'oppose pas tant à un idéal spirituel qu'à un idéal esthétique - celui d'une beauté formelle que l'écrivain associe plutôt à des corps élastiques, souples et bondissants. Quant aux danseuses, ce n'est plus l'excès musculaire, mais bien le manque de chair, imputable à une maigreur excessive, qui est visé :

Je ne sais si vous vous êtes avisé de faire une étude spéciale du cou et de la poitrine d'une danseuse; les clavicules éclairées en dessous font une horrible saillie transversale où viennent s'attacher, comme des cordes de violon sur leur chevalet, quatre à cinq nerfs tendus à rompre, sur lesquels Paganini aurait joué facilement un concerto. ${ }^{15}$

L'œil du critique, assimilable au scalpel d'un chirurgien, dissèque et met à nu la danseuse, dont il ne perçoit plus que l'apparence décharnée, et qui prend, au travers de la comparaison avec Paganini, une dimension fantastique et macabre, sinon diabolique. Les caricatures de danseurs prolongent la critique du corps-machine. Elle donnent à voir à leur tour des corps dont la part mécanique est outrageusement exhibée, à l'instar de l'anatomie. Une caricature de Pigal, parue dans Le Charivari, montre ainsi un danseur aux membres disproportionnés, dont la musculature hypertrophiée contraste avec l'efféminement général de l'allure, et qui paraît comme tiré en l'air par des fils. 
Fig. 1

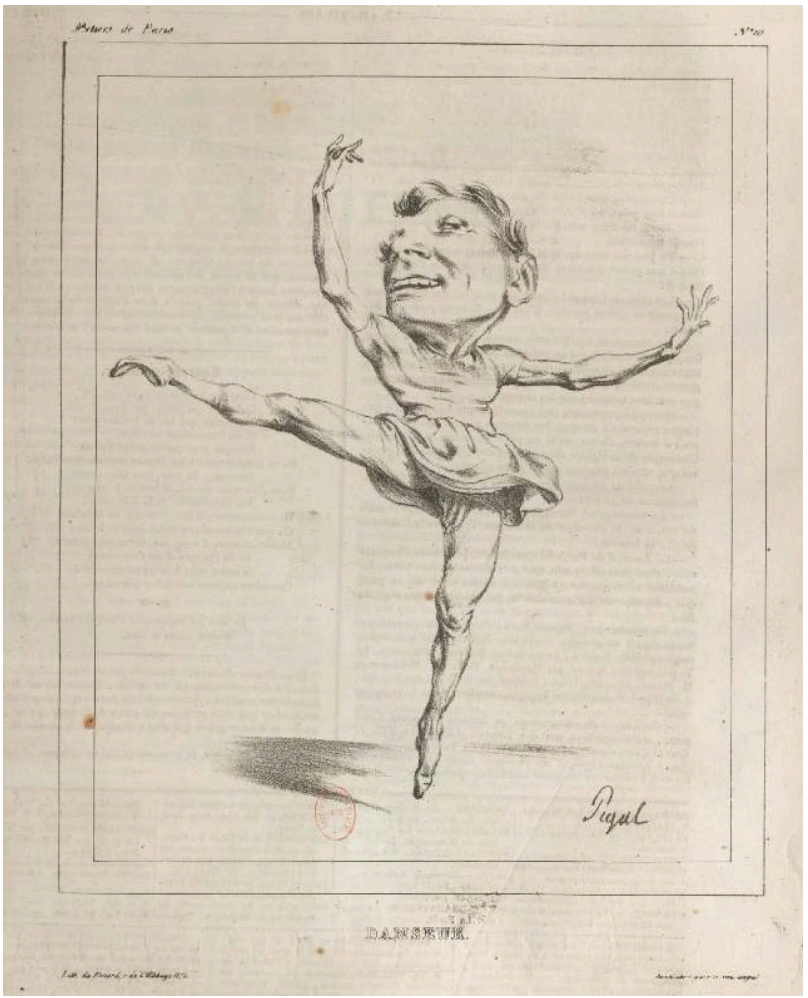

Edmé-Jean Pigal, « Danseur », « Métiers de Paris », n 10, Le Charivari, 1 ${ }^{\text {er }}$ novembre 1833 Source Gallica, BnF, domaine public

La maigreur des mains, aux doigts bizarrement écartés, le cou-de-pied à la cambrure excessive évoquent par ailleurs une créature grotesque, comme échappée d'une danse macabre. Le glissement du registre satirique au registre fantastique ou macabre, qui est aussi un glissement du rire à la crainte - celle que suscite le corps -, apparaît tout à fait explicite chez Grandville, dont l'ouvrage Un autre monde exploite, dans le contexte d'une fantasmagorie, un imaginaire scientifique. Dans le chapitre intitulé «Le royaume des marionnettes ", Grandville représente une danseuse au corps hybride, pourvue d'ailes de libellule et de bras de squelette, souriant complaisamment et mécaniquement au public [fig. 2] - ce dernier détail est du reste souvent la cible des critiques. Quand le danseur exhibe une musculature hypertrophiée, obscène par excès de force, la danseuse s'affiche de son côté comme une créature aux os saillants, par excès de maigreur. 
Fig. 2

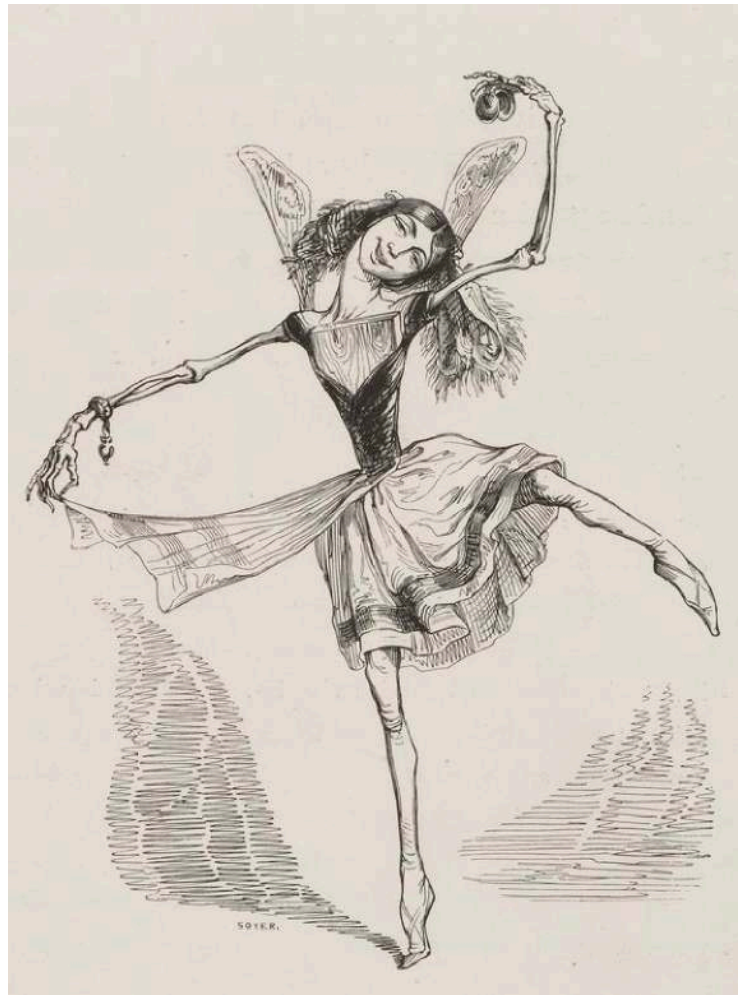

Illustration tirés de l'ouvrage de Jean-Jacques Grandville [texte par Taxile Delord], Un autre monde: transformations, visions, incarnations, ascensions, locomotions, explorations, pérégrinations, excursions, stations..., Paris, H. Fournier, 1844

Source Gallica, BnF, domaine public

On note aussi, dans cette série d'illustrations, le goût pour l'entomologie, qui conduit à figurer les danseurs sous les traits d'insectes, eux-mêmes identifiés à des toupies à ressort - nouvelle variation autour de l'imaginaire de la machine [fig. 3-4]. 
Fig. 3

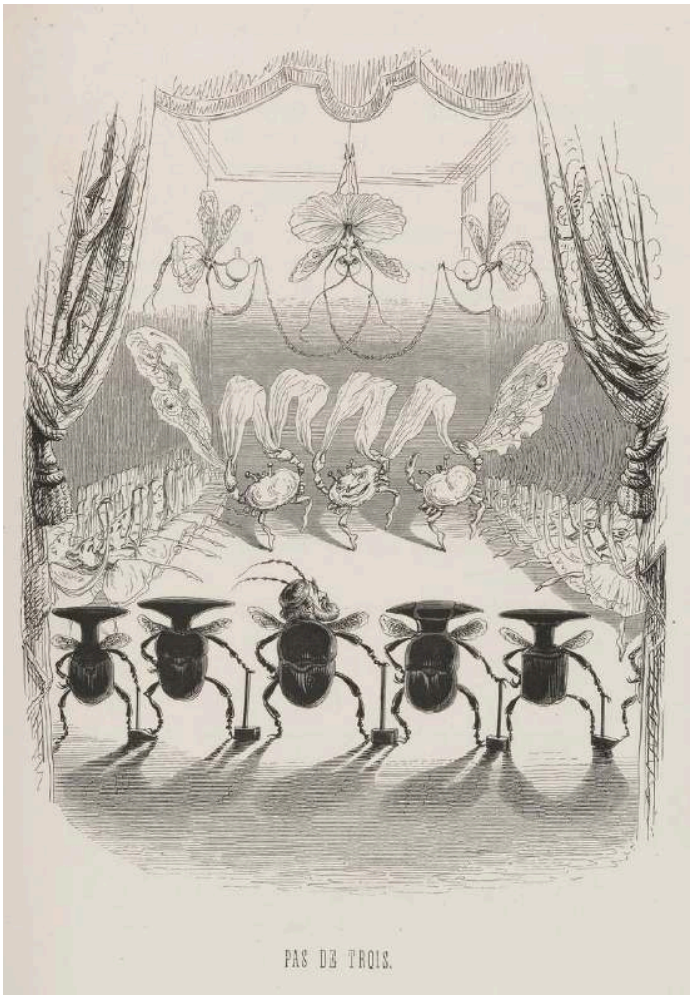

Illustration tirés de l'ouvrage de Jean-Jacques Grandville [texte par Taxile Delord], Un autre monde: transformations, visions, incarnations, ascensions, locomotions, explorations, pérégrinations, excursions, stations..., op. cit.

Source Gallica, BnF, domaine public 
Fig. 4

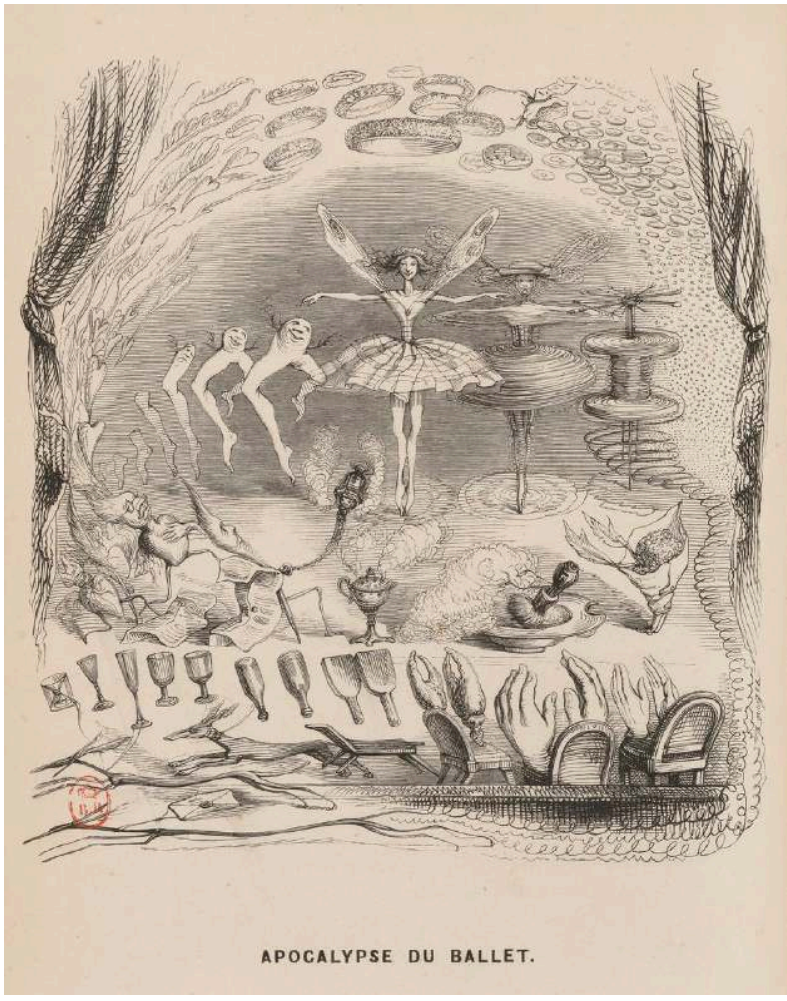

Illustration tirés de l'ouvrage de Jean-Jacques Grandville [texte par Taxile Delord], Un autre monde: transformations, visions, incarnations, ascensions, locomotions, explorations, pérégrinations, excursions, stations..., op. cit.

Source Gallica, BnF, domaine public

17 La métaphore est également reprise dans le dessin de «deux danseurs à ressorts articulés $»^{16}$, associés dans un pas de deux - littéralement mécanique [fig. 5] -, figurant deux pantins désexualisés, au sourire figé : leurs articulations sont soulignées par des vis, tandis que l'écart forcé des jambes évoque l'ouverture d'un compas. 
Fig. 5

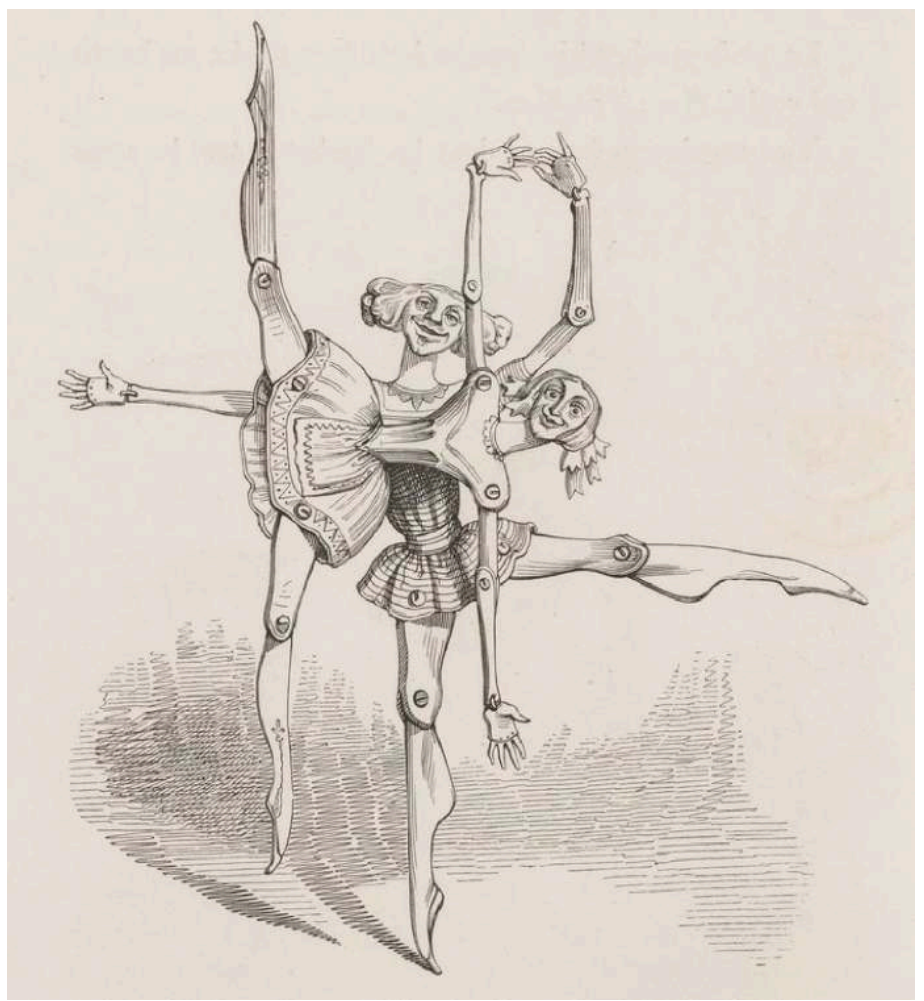

Illustration tirés de l'ouvrage de Jean-Jacques Grandville [texte par Taxile Delord], Un autre monde transformations, visions, incarnations, ascensions, locomotions, explorations, pérégrinations, excursions, stations..., op. cit.

Source Gallica, BnF, domaine public

Le dessin rappelle par ailleurs une image récurrente chez Gautier, reprise dans plusieurs textes, évoquant une danseuse qui « tourne sur l'ongle de son orteil avec une jambe parallèle à l'horizon dans la gracieuse attitude d'un compas forcé $»^{17}$. L'analogie géométrique ne cible donc pas seulement le danseur, figure traditionnellement honnie dans les discours de l'époque, mais bien le corps dansant en général. On voit dès lors que ce qui condamne l'artiste, homme ou femme, c'est à la fois la disproportion et l'exhibition - celle des muscles, des os, des articulations, des jointures -, qui sont autant de signes d'une mécanique à l'œuvre, dont on ne sait, de Dieu ou du diable, qui l'anime. Exagérés par la caricature, ces détails contribuent ainsi à mettre au jour tout un ensemble de choses cachées, normalement situées en périphérie de la représentation: le travail technique, l'effort physique et musculaire, la dépense d'énergie, tout ce que l'art véritable - l'illusion sur laquelle repose le corps romantique - se doit a contrario de dissimuler au spectateur.

\section{Du « dressage » des corps dansants}

Ces choses destinées à rester cachées des yeux du public, la littérature du XIX siècle s'y intéresse toutefois de près dans une perspective non plus seulement polémique et prescriptive, mais documentaire et démystificatrice, en utilisant la science comme modèle d'observation, d'investigation et d'analyse d'une réalité sociale - celles-ci passant par ce qu'on pourrait appeler un "état des corps ». Les années 1840, qui 
coïncident avec un épanouissement - qui est déjà, pour certains commentateurs, un " déclin »- du ballet romantique, voient en effet se manifester un intérêt nouveau pour les coulisses des théâtres, à commencer par celles de l'Opéra, dont la fréquentation est un privilège acquis aux abonnés depuis les débuts de la monarchie de Juillet et le changement de statut de l'Académie royale de musique, dont la direction est alors confiée à un "directeur-entrepreneur ». Le roman, la presse et l'iconographie satirique exploitent largement l'imaginaire licencieux attaché à ces arrière-scènes, notamment au foyer de la danse, devenu un lieu de sociabilité mondaine et galante. Propices aux intrigues, les arrière-scènes sont toutefois aussi dépeintes comme le lieu de la fabrique de l'illusion - «l'envers de la féerie contemporaine $»^{18}-$, le lieu où se révèle au regard de l'abonné ou du flâneur de passage un corps trivial et non-spectaculaire, celui de la danseuse au travail.

De nombreuses publications, apparentées au genre hybride de la physiologie, qui connaît une grande popularité dans les années 1840, témoignent d'une curiosité particulière pour ces mondes cachés. Elles s'emparent du sujet à leur manière, plaisante et souvent ironique, en adoptant une approche pseudo-scientifique à mi-chemin du reportage et de la fiction documentaire ${ }^{19}$. À ce corpus il convient d'intégrer aussi bien des physiologies stricto sensu - ouvrages de petit format, divisés en courts chapitres, parfois illustrés - portant sur les foyers et les coulisses des théâtres, comme celles de Jacques Arago $^{20}$ ou d'Albert Vizentini ${ }^{21}$, que des articles consacrés à des types sociaux insérés dans des ouvrages plus conséquents ou de nature encyclopédique, à l'exemple de ceux de Gautier sur le rat, publié dans Les Français peints par eux-mêmes ${ }^{22}$, ou de Louis Huart, figurant dans le Muséum parisien ${ }^{23}$. Tous ces textes participent d'un travail global de recension, de classification et d'analyse des espèces sociales, qui se place sous l'influence, traitée de manière fantaisiste ou parodique, de Buffon et des sciences naturelles.

21 De ces enquêtes émerge une figure, ou plutôt un type, lié à un milieu - au sens à la fois biologique et social -, celui du rat - plus largement celui de la danseuse de corps de ballet -, dont le corps, ignoré le plus souvent des chroniques de spectacle, apparaît comme un contrepoint réaliste au corps fantasmatique de l'étoile. L'image de la machine, contre-modèle dans la représentation scénique des corps, devient dans ce contexte nouveau l'image du travail quotidien mené dans la salle de danse, espace associé à l'idée de contrainte, à la différence du foyer des abonnés et des coulisses en général, affectés d'une dimension frivole ou licencieuse. Ces enquêtes passent toutes par un topos, celui de la classe quotidienne, destinée à façonner un corps exercé à répéter inlassablement les mêmes gestes. À la répétition mécanique des actions triviales du quotidien, comme le lever à heure fixe, l'habillage et le déshabillage permanents, les déplacements renouvelés du domicile au théâtre et du théâtre au domicile dans une capitale moderne de la taille de Paris ou de Londres, s'ajoute celle d'une grammaire complexe de pas, répertoriés dans les nomenclatures de la danse académique :

Le maître fait exécuter des assemblés, des jetés, des ronds de jambes, des glissades, des changements de pied, des taquetés, des pirouettes, des ballons, des pointes, des petits battements, des développés, des grands fouettés, des élévations et autres exercices gradués selon la force des élèves : toutes font le pas ensemble et viennent ensuite le refaire devant le professeur trônant gravement entre deux chaises [...]. ${ }^{24}$ déleste les individus de leurs particularismes, justifiant par là la récurrence de la 
métaphore militaire dans les descriptions. Le Britannique Albert Smith, auteur de plusieurs physiologies portant sur des types sociaux, parmi lesquelles on compte une histoire naturelle de la danseuse ${ }^{25}$, parle ainsi des danseuses comme d'une "armée de guerrières " [ "an army of Female Warriors $\left.{ }^{26}\right]$ et son récit, qui se rattache au genre biographique, s'ouvre sur une illustration représentant un maître de ballet dirigeant une troupe de danseuses portant le fusil à l'épaule. La représentation des corps en plein travail révèle à cet égard le paradoxe inhérent à la danseuse. Derrière une apparente délicatesse, qui confine à la fragilité, se cache une machine corporelle d'une puissance phénoménale: «des jambes de fer " et "des membres d'acier ${ }^{27}$, souligne Albert Vizentini dans sa Physiologie des foyers.

Le dressage collectif est parallèlement associé à la violence et à la contrainte exercées sur les corps, parfois renforcées par le recours à un outillage technologique sophistiqué. Pour atteindre l'idéal - la norme imposée par la scène -, les corps doivent, chaque jour, se tordre, se casser, se disloquer, se briser - une terminologie usuelle dans ces discours, qui renvoie à un entraînement ritualisé et codifié :

Tirer sur les barres, se faire monter sur les genoux, les pliés, les écarts, se renverser jusqu'à ce que tous les membres craquent en mesure, s'éreinter, s'époumoner sans cesse, n'écouter ni fatigue ni paresse, sont les gammes quotidiennes de la danseuse. ${ }^{28}$

Des instruments de travail - le terme de coercition serait plus adéquat -, comme la barre, sont par ailleurs prévus pour aider à assouplir et à redresser les corps défaillants :

Le long des murs sont plantés des crampons de fer et des traverses de bois, dont il serait difficile à un bourgeois naïf de deviner la destination, et qui ont de vagues ressemblances avec les instruments de torture et les chevalets d'estrapade du Moyen Âge ; n'était la bonne et honnête figure du professeur tranquillement assis sa pochette à la main, l'on ne serait pas trop rassuré. [...]

[...] dans les intervalles, elles [les élèves] vont se pendre aux crampons pour exécuter des pliés, et s'exercent à faire des arabesques en jetant leurs jambes sur ces traverses de bois dont nous avons parlé tout à l'heure. Elles restent ainsi le pied à la hauteur de l'épaule dans une position impossible qui tient le milieu entre la roue et l'écartèlement. ${ }^{29}$

En outre, la danseuse "doit mettre ses pieds dans les boîtes $»^{30}$, toujours selon les termes de Gautier, qui précise à cet égard qu' « une heure de cet exercice équivaut à six lieues avec des bottes fortes dans les terres labourées par un temps de pluie $»^{31}$. Ces fameuses boîtes font partie de l'attirail technologique mis en place par la pédagogie institutionnelle et visant à assouplir les tendons, étirer les muscles et, sinon à améliorer les performances, du moins à modifier les corps, en l'occurrence à favoriser l'en-dehors des pieds et des jambes, sur lequel se fonde la danse académique. Ce détail réaliste, particulièrement frappant pour un lecteur naïf ou ignorant des réalités de la danse académique, renvoie au développement, exactement contemporain de ces écrits, des pratiques orthopédiques ${ }^{32}$. L'objet, loin d'être appréhendé sous l'angle technologique dans les écrits sur la danse, est alors exploité par l'iconographie satirique, comme en témoigne une illustration de la série d'Édouard de Beaumont, L'Opéra au XIX siècle, que publie Le Charivari [fig. 6]. 
Fig. 6

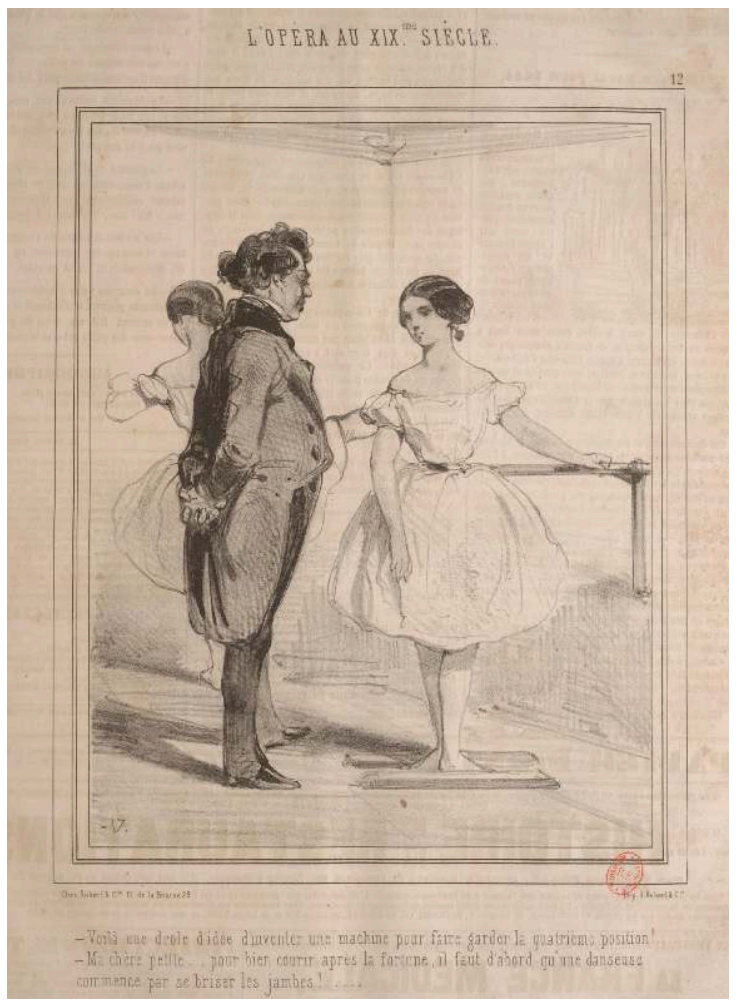

Édouard de Beaumont, "L'Opéra au xıx siècle », n 12, Le Charivari, 9 septembre 1844 Texte sous l'illustration:

- Voilà une drôle d'idée d'inventer une machine pour faire garder la quatrième position !...

- Ma chère petite... pour bien courir après la fortune, il faut d'abord qu'une danseuse commence par se briser les jambes !...

Source Gallica, BnF, domaine public

Dans ces descriptions, la machine, née du progrès technique et censée aider au perfectionnement de cette autre machine qu'est le corps, apparaît avant tout comme l'instrument d'une déshumanisation. La classe de danse, avec son décor claustral et ses pratiques contre-nature, a de fait plutôt l'air de ressusciter l'Inquisition et l'usage ancien de la question que de mettre en œuvre des pratiques novatrices, relevant d'un progrès entendu comme bénéfique aux individus. Charles de Boigne, auteur des Petits Mémoires de l'Opéra, autre ouvrage de démystification des arrière-scènes, recourt ainsi à une anecdote exemplaire, souvent reprise dans les récits sur les coulisses de l'Opéra, montrant les sévices auxquels la danseuse se soumet dans le but de "redresser " son corps :

Une danseuse dévorée d'ambition, mademoiselle X..., avait inventé [...] une manière fort ingénieuse de se casser et de se tourner en même temps [...].

Mademoiselle X... se couchait par terre, le visage collé contre le plancher, les jambes étendues horizontalement; puis elle faisait monter et peser sur elle sa femme de chambre. ${ }^{33}$

Le motif de la fatigue, associée à quelques autres symptômes, comme la maigreur, beaucoup moins liée dans ces écrits à une contrainte esthétique que l'on s'impose qu'à la pauvreté, aux privations et à une mauvaise alimentation, vient conforter le discours de "vérité » associé à l'exploration des coulisses. Il est notamment souvent question de l'« après ", ce moment où les corps se révèlent dans leur trivialité. Après la classe, la danseuse est, selon Gautier, « haletante, trempée de sueur, les pieds endoloris », tandis 
qu'après la répétition, elle a les "membres brisés de fatigue ", image qui renvoie à celle du cheval après la course. La danseuse, à l'instar de l'animal, donne ainsi à voir un corps qui se tient les côtes, qui souffle, qui ruisselle, qui tousse, qui crache, qui se mouche, qui s'enveloppe dans des pelisses, si elle ne doit pas retravailler, et qui, si elle doit encore danser, s'éponge à grand renfort de loques, boit force verres de bouillon, bouffe sa jupe, fait son chausson, et, dès que le tout est sec, repart après s'être mis du rouge sur les lèvres, du blanc sur les épaules, et un nouveau sourire sur la figure. ${ }^{34}$

Ces représentations d'un corps malade, usé, dépoétisé sans pour autant être dégradé à la manière des descriptions que l'on trouve dans le roman naturaliste, semblent indiquer qu'il y a quelque chose de pathologique dans le travail de la danse, manière de renouer avec un préjugé ancien regardant l'art chorégraphique. Par-delà la description d'un corps littéralement épuisé, c'est aussi toute la dialectique du proche et du lointain, au cœur de l'imaginaire romantique de la danse, qui est ici mise en scène. L'idéalisation naît de l'éloignement, quand la proximité contribue à démythifier le corps dansant.

\section{Du bon usage de la machine : donner une « base positive » à l'art chorégraphique}

Ce quotidien, répétitif et harassant, vécu par les corps dansants, est associé de surcroît à un milieu hermétiquement clos, coupé des bruits et des activités de la ville, qui peut s'apparenter aussi bien à la prison, à l'usine, à l'hôpital qu'au monastère. Il permet ainsi de comprendre l'analogie puissante qui se dessine entre la danseuse, ressaisie dans son effort quotidien, et l'ouvrière - une ouvrière en quelque sorte martyre de son art. On songe à Degas, qui, avant de peindre les danseuses, s'était intéressé aux chevaux de course - au corps de l'animal en plein effort - et à différentes catégories d'ouvrières au travail - repasseuses, modistes ou blanchisseuses. Les physiologies font par ailleurs bien percevoir la parenté entre la figure de la danseuse - qu'elle soit rat, marcheuse ou figurante - et celle de la grisette, personnage familier du roman du XIx ${ }^{e}$ siècle et objet, elle aussi, de l'observation attentive des physiologistes ${ }^{35}$. L'analogie entre ces vies minuscules, souvent ternies par le malheur, et ces corps, usés quotidiennement par l'effort et le manque, vient rappeler que ces évocations, certes en partie fantasmées, sur lesquelles se greffe un imaginaire à la fois social et religieux, coïncident avec les débuts de la société industrielle, laquelle va de pair avec une valorisation de l'efficacité - du rendement - et un nouveau contrôle opéré sur les gestes ${ }^{36}$. L'entraînement des corps dansants, dans le cadre d'une institution telle que l'Académie royale de musique, emblème de la bourgeoisie sous Louis-Philippe comme sous le Second Empire, relève ainsi, avec tous les guillemets qui s'imposent, d'une forme de "fabrique industrielle " des corps - ceux destinés à former l'armée dansante, redoutablement efficace, qu'on appelle le corps de ballet -, dont l'anonymat et la ressemblance cultivée des membres viennent s'inscrire en contrepoint du corps singulier de l'étoile. Cette dernière, qui s'impose d'abord par son nom, véhicule en effet un imaginaire social tout différent.

Les représentations du travail de la danse soulèvent la question du traitement conféré à cette "machine ", à cette mécanique complexe qu'est le corps dansant, et ouvrent dès lors sur des enjeux pédagogiques. Dans ses travaux, qui portent plus spécifiquement sur les gymnastes, Georges Vigarello montre à cet égard comment le $\mathrm{XIX}^{\mathrm{e}}$ siècle voit émerger de nouvelles pédagogies du corps, fondées sur les progrès des connaissances anatomiques et médicales, qui visent à améliorer les performances physiques. Le 
tableau du dressage des corps, esquissé par les physiologies, dont la veine pseudoscientifique participe tout autant de la satire sociale que du fantasme autour de ces mondes secrets que sont les coulisses des théâtres, trouve à ce titre une forme de prolongement savant, délesté de considérations moralisantes, dans les discours des pédagogues de la danse des années 1850 comme Arthur Saint-Léon et Léopold Adice. Saint-Léon, danseur, chorégraphe et maître de ballet de renom - de Paris à SaintPétersbourg -, est l'auteur d'un opuscule intitulé De l'État actuel de la danse et l'inventeur d'un système de notation du mouvement chorégraphique, la sténochorégraphie ${ }^{37}$. Danseur à la carrière plus obscure, devenu professeur de la classe de perfectionnement de l'Académie impériale de musique ${ }^{38}$, Adice est quant à lui l'auteur d'une Théorie de la gymnastique de la danse théâtrale ${ }^{39}$. Si leurs écrits reprennent le leitmotiv selon lequel l'art ne naît que de la contrainte, souvent confondue avec la souffrance du corps, ils s'affranchissent toutefois des fantasmes doloristes et du déterminisme social qui parcourent la littérature des coulisses et font de la danseuse professionnelle non le produit d'un processus vocationnel et élitaire, mais avant tout la victime d'une société dominée par la bourgeoisie - voire la victime expiatoire d'un certain ordre moral si l'on se place dans une perspective religieuse.

31 Ces publications sont bien entendu à replacer dans le cadre d'un double processus, celui d'une autonomisation de la danse, entamée depuis le XviII siècle, et celui d'une légitimation de cet art, demeuré à plusieurs titres en retrait des autres arts de la scène. Ils partent initialement d'un même constat, celui du déclin de la danse française et du corps de ballet de l'Opéra ${ }^{40}$ - poncif qui parcourt les écrits sur la danse dès les années 1840. Ce «déclin» tient d'abord selon eux à une déliquescence de l'enseignement académique, dont la conséquence est la baisse du niveau technique, des danseurs en premier lieu - du fait de leur effacement relatif de la scène -, mais aussi des danseuses du fait du vedettariat, qui tend à sacrifier la qualité du corps de ballet féminin au profit de l'étoile. Ce déclin de la technique ("les classes ont dégénéré41 », écrit explicitement Adice), et donc d'un certain formatage des corps par rapport à la norme académique, généralise la paresse, la négligence et recouvre donc aussi une décadence du corps social - des mœurs donc.

Adice et Saint-Léon apportent à cette question une réponse pédagogique globale, au travers d'un programme qui vise à régénérer le corps dansant et, par là même, une profession, dont Adice ne dissimule pas par ailleurs la grande difficulté, le caractère contraignant et profondément contre-nature - prônant à ce titre une sélection sévère des corps les plus aptes à la soutenir. Il souhaite dans ce contexte conférer à la danse théâtrale "une base théorique raisonnée $»^{42}$, «une base positive $»^{43}$ ou encore "une théorie fondamentale du mécanisme $»^{44}$, présentée comme inédite. De manière intéressante, la discipline chorégraphique, qui repose essentiellement sur une tradition orale, à la différence des autres arts imitateurs, n'est plus nommée «l'art de Terpsichore $\aleph^{45}$ comme il était d'usage dans les traités anciens, mais « la gymnastique de la danse ", expression qui met l'accent sur l'entraînement des corps. Cette appellation nouvelle l'autorise dès lors à se démarquer de conceptions classiques dont il se réclame par ailleurs, comme celles de Blasis, qui privilégie l'analogie classique de la danse avec les beaux-arts, ou de Noverre, qui s'intéresse moins à la « leçon » qu'à la " composition $»^{46}$, autrement dit au résultat scénique. Saint-Léon insiste lui aussi sur la nécessité pour la danse d'adopter une grammaire normative - écrite -, afin de substituer à l'«habileté souvent contestable du professeur $~^{{ }^{47}}$ la mécanique d'une pédagogie raisonnée. Il promeut à cette fin la généralisation de son système de notation 
du mouvement, la sténochorégraphie, qui combine un système de portées musicales et un alphabet de signes et de chiffres :

Pour établir une école de ce genre, seul moyen d'empêcher la ruine de la danse et d'améliorer la position éphémère du professorat, il fallait avant tout créer une langue spéciale, figurée, écrite et remplaçant la parole; pour démontrer les différents mouvements du corps, des bras et des jambes et la durée de ces mouvements, il fallait des signes de convention et des notes; cette langue existe aujourd'hui et nous l'avons nommée la Sténo chorégraphie; elle est à la portée de tous et des essais faits sur des enfants, en ont donné la preuve ; mais par elle-même elle ne peut avoir aucune importance, si elle n'est point généralisée et adoptée dans un conservatoire central. Ce grand point obtenu, il surgira de cette adoption propagée des règles, des méthodes, des exercices, des résumés d'expérience, et du point de vue de l'art, des garanties d'avenir pour le maître comme pour l'élève. ${ }^{48}$

Sans entrer dans le détail «technique ", précisons que ces pédagogies reposent sur une analyse et une décomposition du mouvement ${ }^{49}$ et mettent l'accent tout à la fois sur la répétition du geste et la discipline collective. Elles ne sont pas sans évoquer à cet égard

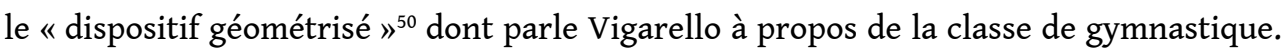
Dans cette configuration, qui renvoie à un idéal mathématique déjà prôné par Carlo Blasis dans son Traité sur la dans $e^{51}$, la métaphore militaire, à laquelle recourt SaintLéon, ne vise pas l'effet pathétique; elle n'a qu'une valeur pédagogique ou méthodologique, comme le souligne la référence du théoricien à Horschelt, chorégraphe viennois fondateur en 1815 d'une troupe de ballet d'enfants, réputée pour ses ensembles sophistiqués, à la discipline impeccable [fig 7] :

M. Horschelt [...] s'appliqua à inculquer à ses jeunes élèves la précision des mouvements, la formation des lignes, des carrés, des ronds, des obliques par une méthode à lui ; elle consiste en manœuvres spéciales, et pour ainsi dire militaires à l'exemple des exercices nombreux et constants qu'on impose aux troupes. ${ }^{52}$

Fig. 7

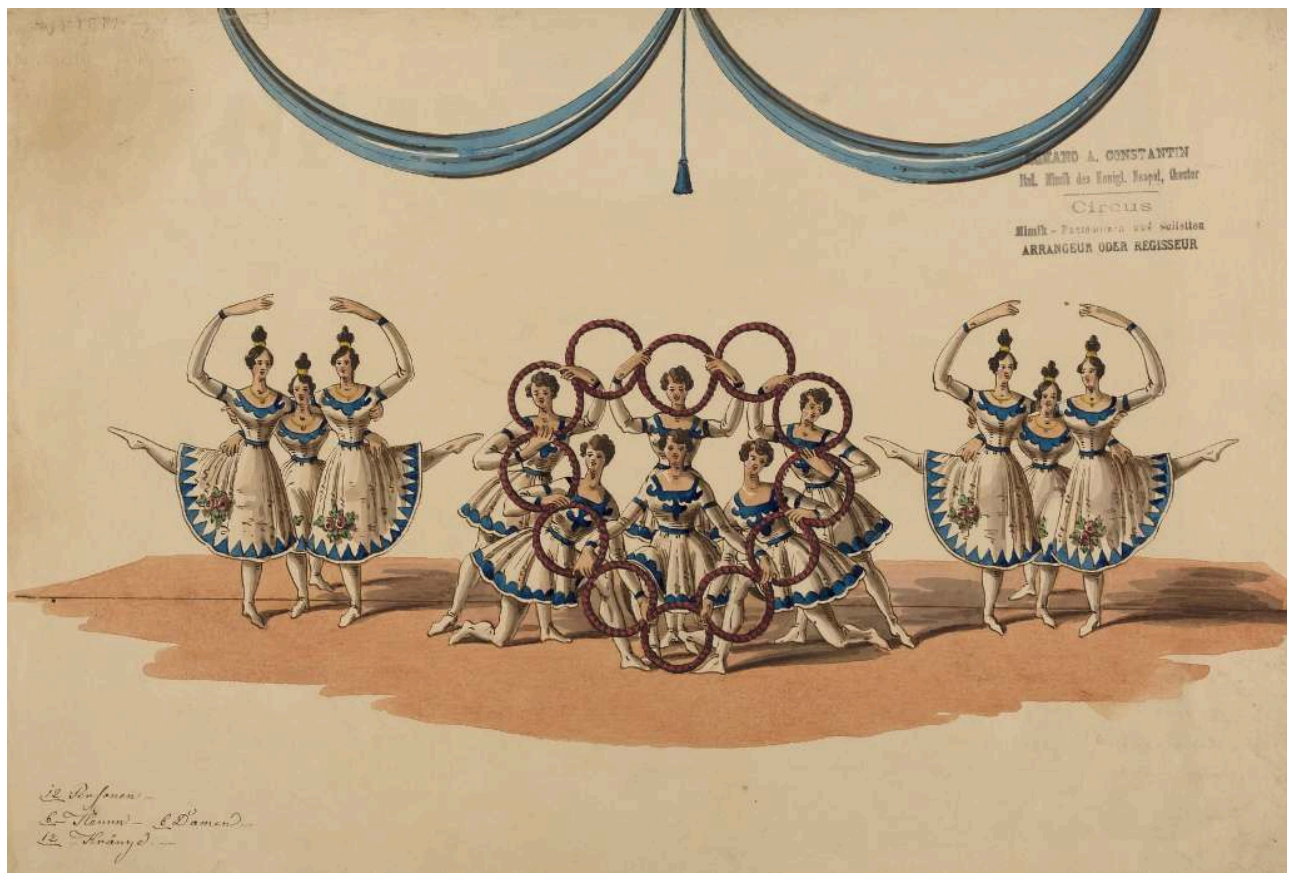

Friedrich Horschelt, Der Berggeist, vers 1818, George Chaffee collection of theatrical caricatures, costume design, scenography, and portraits, Houghton Library, Harvard University. [en ligne] https:// iiif.lib.harvard.edu/manifests/view/drs :43796847 \$ $1 \mathrm{i}$ 

l'ensemble, ce décor indispensable à tout ballet d'action de l'époque, insistent toutefois sur la nécessité d'une instruction complète du danseur : il s'agit certes d'entretenir et de faire fonctionner au mieux la machine corporelle, assimilée ainsi à un moteur image derrière laquelle se profile tout un imaginaire énergétique -, mais pas de couper celle-ci de la nécessaire formation de l'esprit. Saint-Léon comme Adice évoquent à cet égard l'importance de l'apprentissage du dessin, de la musique - disciplines classiques naturellement liées à l'enseignement de la danse académique -, mais aussi et surtout de la science anatomique, chez l'élève comme chez le professeur :

Cependant l'anatomie du corps humain est complètement ignorée de presque tous nos professeurs ; ils croient qu'elle leur serait inutile, et ils n'imaginent pas qu'ils se sont privés d'une ressource immense pour expliquer les mouvements, prévenir les dangers et faciliter et accélérer les progrès, en négligeant d'étudier le jeu des os, des ligaments, des muscles, des nerfs, des fibres qui fonctionnent dans cette gymnastique..$^{53}$

On perçoit là l'imprégnation scientifique de ces pédagogies, peu nouvelles en réalité sur le fond, qui manifestent, en liaison avec l'intérêt marqué pour l'étude de l'anatomie, des préoccupations hygiéniques et sanitaires s'inscrivant pleinement dans un air du temps de plus en plus sensible à ces questions, très largement documentées par la recherche en histoire sociale et culturelle ${ }^{54}$. La deuxième partie du traité d'Adice, intitulée "Monographie des malaises qui résultent de la gymnastique de la danse théâtrale ", propose ainsi un exposé précis, appuyé sur un savoir médical - et approuvé du reste par une autorité médicale -, des différents malaises et traumatismes subis par les corps dansants (la courbature, la crampe, l'accrochement sciatique, la douleur au torse, l'entorse, le point de côté, etc.). Si l'on ne saurait parler ici, sans anachronisme, de «bien-être " du danseur, comme on peut le faire aujourd'hui où la pratique professionnelle de la danse est parfois médicalisée jusqu'à l'outrance, la volonté affichée de prendre soin de la « machine » corporelle, dans le but à la fois d'en prévenir les maux, de la soigner et de la rendre plus performante, n'est pas anodine dans un contexte culturel où le corps dansant reste avant tout un objet de fantasmes.

L'image, ancienne, du "corps-machine », qui nous a servi de point de départ, vient accompagner, au milieu du XIX ${ }^{\mathrm{e}}$ siècle, la représentation inédite du corps dansant au travail, représentation qui s'enrichit ainsi, dans un contexte marqué par l'industrialisation, d'une dimension sociale. Les discours des maîtres de ballet autour de la double question du déclin et de la régénération de la danse, sans rompre avec les conceptions dualistes de leurs prédécesseurs, permettent toutefois de percevoir le caractère en partie fantasmatique des représentations offertes par les écrits sur les coulisses, qu'on retrouve du reste dans les tableaux de Degas. Ces discours manifestent l'évolution du regard sur le corps et témoignent notamment d'un souci nouveau de sa santé et de son efficacité - une efficacité bien ordonnée. La période examinée, située avant le grand tournant naturaliste, reste toutefois dominée par une approche idéalisante. La grande affaire des écrivains-balletomanes, c'est en effet l'illusion suscitée par la représentation scénique, vécue comme un rêve, dont l'héroïne principale est la ballerine. L'imaginaire scientifique, dès lors marginalisé, vient dans ce cadre révéler les ambivalences du corps dansant, toujours partagé entre sa part matérielle, biologique, et sa quête, ambiguë elle aussi, de transcendance. Il nourrit ses 
représentations en creusant notamment le fantasme d'une corporalité idéalisée. Pour autant, le corps dansant, biologisé ou mécanisé, peut aussi devenir, dans une certaine mesure, objet de fascination, au travers notamment du thème de la virtuosité et de la prouesse technique, que nous avons ici laissé de côté. Ce n'est pas un hasard s'il se fait in fine lui-même motif chorégraphique, comme en témoigne notamment Coppélia, ballet de 1870 chorégraphié par Arthur Saint-Léon, qui met en scène (et en abyme), par un retournement ludique de la mythologie romantique, une ballerine incarnant, justement, une poupée mécanique.

\section{NOTES}

1. La Dansomanie, ballet-pantomime en deux actes de Pierre Gardel, musique de Méhul, créé à l'Opéra de Paris le 14 juin 1800.

2. Giovanni-Leopold Adice, Théorie de la gymnastique de la danse théâtrale, avec une monographie des divers malaises qui sont la conséquence de l'exercice de la danse théâtrale : la crampe, les courbatures, les points de côté, etc.., Paris, Impr. de N. Chaix, 1859-1870, p. 14

3. Jean Starobinski, Portrait de l'artiste en saltimbanque, Paris, Gallimard, 2004 [1970], p. 24.

4. Jean-Georges Noverre, Lettres sur la danse, et sur les ballets, Lyon, chez Aimé Delaroche, 1760.

5. L'un des plus célèbres mécanistes du $\mathrm{XVIII}^{\mathrm{e}}$ siècle est Jacques de Vaucanson, qui inventa plusieurs automates humains, comme le joueur de flûte et le joueur de tambourin, et un automate zoomorphe, le fameux canard mécanique dont on pouvait voir les entrailles. Voir à ce sujet les riches analyses de Laurent Guido, « Modèles et images de la danse(use) mécanique, des automates à l'être "électro-humain», dans Laurence Schifano (dir.), La Vie filmique des marionnettes, Nanterre, Presses universitaires de Paris Nanterre, 2008, p. 107-125. [en ligne] https://books.openedition.org/pupo/812; du même auteur, "Vers l'être "électro-humain": dispositifs visuels de la danseuse mécanique aux $\mathrm{xIX}^{\mathrm{e}}$ et $\mathrm{xx}^{\mathrm{e}}$ siècles", dans Olivier Asselin, Silvestra Mariniello et Andrea Oberhuber (dir.), L'Ère électrique. The Electric Age, Ottawa, Presses de l'Université d'Ottawa, 2011, p. 155-180. [en ligne] https://books.openedition.org/uop/403.

6. Jean-Georges Noverre, Lettres sur la danse, op. cit., lettre II, p. 27-28. [orthographe et graphies modernisées]

7. Ibid., lettre X, p. 288-289.

8. Heinrich von Kleist, Über das Marionettentheater, Berliner Abendblättern, 12-15 décembre 1810.

9. Charles-François Mazurier (1798-1828) est un mime et danseur français, qui se rend notamment célèbre comme interprète des rôles de Polichinelle et surtout du singe Jocko au théâtre de la Porte Saint-Martin dans les années 1820.

10. Cette appellation est une survivance de la hiérarchie des genres, établie au siècle précédent, qui distingue trois styles de danse, associés à des types de danseurs, définis notamment par des critères morphologiques : la danse noble, la danse de demi-caractère, la danse comique.

11. Journal des Débats, 24 août 1832.

12. Ibid.

13. La Charte de 1830, 18 avril 1837.

14. Ibid.

15. Ibid. 
16. Jean-Jacques Grandville [texte par Taxile Delord], Un autre monde: transformations, visions, incarnations, ascensions, locomotions, explorations, pérégrinations, excursions, stations..., Paris, H. Fournier, 1844, p. 55.

17. La Presse, 28 juillet 1839.

18. L'expression est le titre de la quatrième partie de mon livre, Les Deux Corps de la danse. Imaginaires et représentations à l'âge romantique, Pantin, Centre national de la danse, coll. « Histoires », 2018.

19. Sur les physiologies, voir Nathalie Preiss, Les Physiologies en France au XIX siècle, Étude historique, littéraire et stylistique, Mont-de-Marsan, Éditions InterUniversitaires, 1999 ; Valérie Stiénon, La Littérature des Physiologies. Sociopoétique d'un genre panoramique (1830-1845), Paris, Classiques Garnier, 2012.

20. Jacques Arago, Physiologie des foyers de tous les théâtres de Paris, Paris, chez les marchands de nouveautés, 1841.

21. Albert Vizentini, Derrière la toile (Foyers, coulisses et comédiens). Petites physiologies des théâtres parisiens, Paris, Achille Faure, 1868.

22. Théophile Gautier, "Le Rat », dans Les Français peints par eux-mêmes. Encyclopédie morale du XIXe siècle, Paris, Léon Curmer, 1841, t. III, p. 248-256.

23. Louis Huart, «Le Rat », dans Muséum parisien. Histoire physiologique, pittoresque, philosophique et grotesque de toutes les bêtes curieuses de Paris et de la banlieue. Pour faire suite à toutes les œuvres de M. de Buffon, 350 vignettes par MM. Grandville, Gavarni, Daumier, Traviès, Lecurieux et Henri Monnier, Paris, Bauger et Cie, Aubert, 1841, p. 88-96. On peut également mentionner l'article d'Albéric Second, « Histoire naturelle. Le rat de province », Le Figaro, 29 octobre 1840.

24. Théophile Gautier, op. cit., p. 253.

25. Albert Smith, The Natural History of the Ballet Girl, illustré par A. Henning, Londres, D. Bogue, 1847.

26. Ibid., p. 24.

27. Albert Vizentini, op. cit., p. 27.

28. Ibid.

29. Théophile Gautier, op. cit., p. 253.

30. Ibid.

31. Ibid.

32. Georges Vigarello mentionne à cet égard la «balançoire orthopédique » de Charles Pravaz, mise en place en 1827. Voir « Le corps travaillé. Gymnastes et sportifs au XIXe siècle ", dans Alain Corbin (dir.), Histoire du corps, t. II, De la Révolution à la Grande Guerre, Paris, Seuil, coll. " Points », 2005, p. 337.

33. Charles de Boigne, Petits Mémoires de l'Opéra, Paris, Librairie nouvelle, 1857, p. 35.

34. Adolphe Dupeuty, "L'Intérieur de l'Opéra », Où est la femme?, préface de Jules Noriac, E. Dentu, 1864, p. 83.

35. Louis Huart, Physiologie de la grisette, Paris, Aubert, Lavigne, 1841.

36. Voir Georges Vigarello, op. cit., p. 330-336.

37. Arthur Saint-Léon, La Sténochorégraphie, ou l'Art d'écrire promptement la danse, Paris, 1852 ; De l'État actuel de la danse, Lisbonne, Typographie du Progresso, 1856.

38. Voir Sandra Noll Hammond, «Ballet's Technical Heritage: The Grammaire of Léopold Adice », Dance Research: The Journal of the Society for Dance Research, 1995, vol. 13, n 1, p. 33-58.

39. Giovanni-Leopold Adice, Théorie de la gymnastique de la danse théâtrale, op. cit.

40. Saint-Léon écrit ainsi : "Il résulte de cela que le rat, n'étant qu'un accessoire plus ou moins joli, mais tout à fait incapable, que le figurant étant honni, méprisé et banni, le véritable corps de ballet n'existe plus, qu'il n'y a plus ni ensemble, ni ligne, ni figure, ni effets de masse. » (De l'État actuel de la danse, op. cit., p. 8.)

41. Adice, op. cit., p. 11. 
42. Ibid., p. 6.

43. Ibid.

44. Ibid., p. 7.

45. Ibid., p. 58.

46. Ibid., p. 104.

47. Saint-Léon, De l'État actuel de la danse, op. cit., p. 8.

48. Ibid., p. 9.

49. On retrouve ces principes et ces préoccupations dans des innovations technologiques plus ou moins contemporaines comme la chronophotographie ou le phénakistiscope.

50. Vigarello, op. cit., p. 337.

51. Vigarello mentionne de manière intéressante, dans le chapitre précédemment cité de l'Histoire du corps, ce passage du traité de Blasis : « Si je formais une école de Danse, je mettrais de suite en pratique ce moyen que j'ai imaginé, que je crois essentiellement utile, et que tout professeur, sans être obligé de savoir dessiner, peut employer, c'est-à-dire, que je formerais pour les élèves une espèce d'abécédaire composé de lignes droites, pour toutes les positions de leurs membres, donnant à ces lignes et à leurs combinaisons respectives, les dénominations adoptées par les géomètres, savoir de perpendiculaires, d'horizontales, d'obliques, d'angles droits, aigus, obtus, etc. Langage que je crois même indispensable dans nos leçons, en traçant sur l'ardoise ces figures par des lignes droites, comme dans l'exemple donné ici : cent élèves à la fois, ayant les yeux fixés sur ce modèle, concevront de suite, ensemble, et très facilement, leurs positions, leurs attitudes, sans que le maître soit obligé de s'époumoner par des discours longs à chacun d'eux. En outre de cela, les plus diligents de ces écoliers pourraient copier, sur de petites ardoises, ces mêmes figures, et les emporter, pour les étudier chez eux, comme l'enfant qui commence à syllaber, étudie dans son abécédaire, sans avoir près de lui le maître... et son fouet. (Charles Blasis, Traité élémentaire, théorique et pratique de l'art de la danse, Milan, J. Beati et A. Tenenti, 1820, cité par Georges Vigarello, op. cit., p. 335.)

52. Saint-Léon, De l'État actuel de la danse, op. cit., p. 12.

53. Adice, op. cit., p. 47.

54. Voir par exemple Georges Vigarello, Histoires des pratiques de santé. Le sain et le malsain depuis le Moyen Âge, Paris, Le Seuil, 1999 [1993]; Olivier Faure, Les Français et leur médecine au XIXe siècle, Paris, Belin, 1993 ; Patrice Bourdelais (dir.), Les Hygiénistes, enjeux, modèles et pratiques (XVIII ${ }^{e}-\mathrm{XX}^{e}$ siècles), Paris, Belin, 2001.

\section{RÉSUMÉS}

Cet article se propose d'étudier la circulation d'une image ancienne, celle du " corps-machine », dans divers écrits sur la danse académique, et d'en mettre en lumière les significations et enjeux contradictoires au milieu du XIX ${ }^{\mathrm{e}}$ siècle. Dans le contexte du ballet romantique, l'image définit d'abord un contre-modèle esthétique et moral, dont témoignent la littérature sur les spectacles ou la caricature. Elle rend compte par ailleurs de l'intérêt nouveau porté au travail quotidien de la danse, auquel s'attachent en particulier les physiologies, qui mêlent fantasme et regard pseudo-scientifique sur les corps. Cet éclairage sur les coulisses de la danse trouve un prolongement savant chez certains pédagogues de la danse, soucieux de donner une «base 
positive " à l'art chorégraphique et, ainsi, dans un contexte de déploration autour du " déclin » de la danse, de le « régénérer».

This paper is intended to study the spreading of the older image of the "body machine " in various mid- $19^{\text {th }}$ century writings on academic dance and to highlight its contradictory meanings and issues. In the specific context of romantic ballet, this image first defines an aesthetic and moral counter-model as it emerges from critical reviews or caricatures. Notably in French Physiologies - a literary genre combining fantasy and pseudo-scientific look on bodies - it accounts for the then new interest in daily dance practicing. This glimpse behind the scenes finds a scholarly echo among some dance pedagogues eager to give choreographic art a substantive basis and thus regenerate it even in the context of an alleged « decline » of ballet.

\section{INDEX}

Mots-clés : ballet, danse académique, corps-machine, Opéra de Paris

Keywords : ballet, academic dance, body machine, Paris Opera

\section{AUTEUR}

\section{BÉNÉDICTE JARRASSE}

Université Paris Sorbonne Nouvelle 